\title{
CHIRONOMIDAE (DIPTERA) EM SUBSTRATOS ARTIFICIAIS NUM PEQUENO RESERVATÓRIO: REPRESA DO MONJOLINHO, SÃO CARLOS, SÃO PAULO, BRASIL
}

Leny Célia da Silva Correia(1) \& Susana Trivinho-Strixino(2)

\begin{abstract}
Chironomidae (Diptera) on artificial substrates within a small reservoir: Represa do Monjolinho (São Carlos, São Paulo, Brasil)The present study aimed to verify possible taxonomic and functional differences between the Chironomidae assemblages that colonize organic and inorganic substrates of different structures. Polymers (polystyrene and nylon mesh), minerals (pyro clay and sand) and organic vegetable substrates (wood branches and litter of Scirpus cubensis and Cabomba piauhyensis) were immersed in Monjolinho Reservoir water column in nylon mesh bags. After 30 days, they were taken and washed through a $0.21 \mathrm{~mm}$ mesh sieve. The organisms retained by the sieve were identified under stereoscopic and/or optic microscopes. The 3432 larvae analyzed pointed to the presence of 17 taxonomic units. Goeldichironomus neopictus was the predominant species, colonizing abundantly all the substrates (>73\%). The quantitative (Euclidean Distance) and qualitative (Jaccard Coefficient) cluster analyses pointed to different groups. The former grouped the
\end{abstract}

(1) Programa de Pós Graduação, Recursos Naturais. Tel/Fax: (016) 260-8305. E-mail: lenycl@excite.com; (2) Laboratório de Entomologia Aquática, Departamento de Hidrobiologia, Universidade Federal de São Carlos, Via Washington Luiz Km 235, CEP: 13565-905 São Carlos, São Paulo, Brasil.

Com auxílio da CAPES e do PPG-ERN da UFSCar 
assemblages that had colonized sand and wood branches (substrates with the greatest numeric densities and richness), polymeric substrates (polystyrene and nylon mesh) and macrophyte litters. Jaccard Coefficient pointed to the greatest similarity of the assemblages present on organic vegetable and on mineral substrates. On the other hand, the assemblages were functionally similar because of the high participation of collectors on all the substrates (>95\%).

Key words: Chironomidae, artificial substrates, Goeldichironomus neopictus, taxonomic structure, feeding guilds

\section{Resumo}

O presente estudo teve como objetivo verificar possíveis diferenças taxonômicas e funcionais entre as assembléias de Chironomidae que colonizam substratos orgânicos e inorgânicos estruturalmente distintos. Substratos poliméricos (isopor e tela de náilon), minerais (pedra de argila expandida e areia grossa) e orgânicos vegetais (detritos de Scirpus cubensis e Cabomba piauhyensis; detritos de madeira), contidos em sacos de náilon, foram submersos na Represa do Monjolinho. Estes, após 30 dias, foram retirados, lavados em peneira de malha de $0,21 \mathrm{~mm}$ e os organismos retidos identificados sob microscópios estereoscópicos e/ou óptico. A análise de 3432 larvas indicou a presença de 17 unidades taxonômicas das quais Goeldichironomus neopictus foi predominante, com expressiva participação em todos os substratos (>73\%). As análises de agrupamentos quantitativa (Distância Euclidiana) e qualitativa (Coeficiente de Jaccard) apontaram para diferentes arranjos. A primeira agrupou as assembléias que colonizaram areia e madeira (com maiores densidades numéricas e riqueza de táxons), os substratos poliméricos (isopor e tela de náilon) e os detritos de macrófitas. A segunda indicou maior semelhança entre as assembléias presentes nos substratos orgânicos vegetais e nos minerais. Em contraposição, devido à elevada representatividade de coletores em todos os substratos (>95\%), as assembléias apresentaramse funcionalmente similares.

Palavras-chave: Chironomidae, substratos artificiais, Goeldichironomus neopictus, estrutura taxonomica, guildas de alimentação 


\section{Introdução}

A maioria dos insetos aquáticos apresenta íntima associação com o substrato, pelo menos, durante uma fase de sua vida. Os substratos exercem grande influência sobre os insetos, podendo afetar seu crescimento e sobrevivência, pois Ihes fornecem hábitat, alimento e proteção (Minshall, 1984). Hábitats heterogêneos, contendo diferentes tipos de substratos, propiciam a instalação de uma grande variedade de organismos (Street \& Titmus, 1982), pertencentes a diversos grupos funcionais (Peso, 1995).

Uma ampla variedade de materiais orgânicos e inorgânicos de origem autóctone e alóctone compõem os substratos disponíveis nos sistemas aquáticos. Substratos orgânicos, geralmente, consistem de algas filamentosas, musgo, macrófitas e animais presentes no ambiente aquático, além de materiais vegetais provenientes do ambiente terrestre. Os substratos inorgânicos são, em geral, constituídos de minerais que variam em tamanho, podendo compreender desde argilas microscópicas a grandes pedregulhos. Eles, também, podem incluir objetos produzidos pelo homem, como, por exemplo, garrafas, latas e concreto (Minshall, 1984; Ward, 1992).

A impossibilidade de amostragem dos macroinvertebrados presentes em determinados hábitats através do uso de aparatos de coleta convencionais (dragas e redes), deu origem ao estudo da colonização de substratos artificiais por esta fauna (Cairns, 1982). No presente estudo, optou-se pela utilização de substratos artificiais devido ao fato deste método possibilitar a comparação das assembléias de organismos que colonizam diferentes substratos em um período conhecido. Portanto, o objetivo deste trabalho foi verificar possíveis diferenças taxonômicas e funcionais das assembléias de Chironomidae que colonizam substratos orgânicos e inorgânicos de diferentes estruturas introduzidos em uma lagoa artificial.

\section{Material e Métodos}

O estudo foi desenvolvido na Represa do Monjolinho $\left(47^{\circ} 54^{\prime} \mathrm{W}\right.$ $22^{\circ} 00^{\prime} S$ ), uma pequena lagoa artificial ( $4,69 \mathrm{ha}$ ) de baixa profundidade (máx.: $3 \mathrm{~m}$; média: 1,7 m), localizada no campus da Universidade Federal de São Carlos (São Carlos, SP). 
Foram utilizados como substratos artificiais materiais orgânicos vegetais (detritos de madeira e de duas macrófitas aquáticas, com diferentes velocidades de decomposição - Cabomba piauhyensise Scirpus cubensis), poliméricos (tela de náilon e flocos de isopor) e inorgânicos minerais (pedras de argila expandida e areia grossa). Três réplicas de cada substrato foram introduzidas em sacos de tela de náilon de $20 \times 20$ $\mathrm{cm}$ com malha de 0,6 $\mathrm{mm}$. Em cada saco foi colocado aproximadamente $500 \mathrm{ml}$ de cada material, exceto tela de náilon (1 pedaço de $20 \mathrm{~cm}^{2}$ ) e detritos de macrófitas ( $5 \mathrm{~g}$ de cada vegetal previamente secos em estufa). Estes foram introduzidos na Represa do Rio Monjolinho em 21/11/1997, em um ponto escolhido aleatoriamente, onde ficaram submersos a uma distância de $1 \mathrm{~m}$ do sedimento e 1,5 $\mathrm{m}$ da superfície.

Após um período de 30 dias, tempo considerado ideal para colonização por macroinvertebrados (Cover \& Harrel, 1978; Neiff \& Poi De Neiff, 1990; Stockley et al. 1998), as amostras foram cuidadosamente retiradas, acondicionadas em sacos plásticos e levadas para o laboratório, onde se procedeu à triagem, fixação em álcool a 70\% e identificação sob microscópio estereoscópico e/ou óptico.

As assembléias de Chironomidae presentes nos diferentes substratos foram comparadas taxonomicamente através das análises de agrupamentos quantitativa (Distância Euclidiana) e qualitativa (Coeficiente de Jaccard). Ambas foram realizadas através do método de agrupamento de pares com médias aritméticas não ponderadas (UPGMA).

A estrutura funcional das assembléias foi estabelecida segundo a classificação das guildas de alimentação de Merritt \& Cummins (1984). Foram consideradas as seguintes categorias funcionais: retalhadores, incluindo herbívoros e comedores de grandes partículas orgânicas; coletores, abrangendo filtradores e roçadores de pequenas partículas; raspadores de algas perifíticas; predadores.

\section{Resultados}

A análise de 3432 larvas indicou a presença de 17 unidades taxonômicas (11 Chironominae, 4 Tanypodinae e 2 Orthocladiinae) (Tab. I). Goeldichironomus neopictus foi o táxon predominante, apresentando expressiva participação em todos os substratos (>73\%). 
As análises de agrupamentos apontaram para diferentes arranjos (Figs. 1A - B). A análise quantitativa (Distância Euclidiana) agrupou com maior similaridade as assembléias que colonizaram areia e madeira, os substratos poliméricos (isopor e tela de náilon) e os detritos de macrófitas. A qualitativa (Coeficiente de Jaccard) indicou maior semelhança taxonômica entre as assembléias presentes nos substratos orgânicos vegetais (madeira, $C$. piauhyensis e $S$. cubensis) e nos minerais (areia e pedra).

\section{Tabela I}

Participação relativa (\%) dos táxons de Chironomidae e número total de indivíduos coletados nos diferentes substratos artificiais.

\begin{tabular}{|c|c|c|c|c|c|c|c|c|}
\hline Chironominae & 94,06 & 94,79 & 95,85 & 98,60 & 98,79 & 98,77 & 98,87 & 97,93 \\
\hline Chironomini & 90,10 & 94,79 & 94,62 & 98,10 & 98,57 & 97,78 & 97,74 & 97,17 \\
\hline Polypedium (Asheum) beckae & & 1,04 & 0,77 & 0,90 & 0,22 & 0,25 & 0,38 & 0,55 \\
\hline Chironomus gr. dec. sp. & & & & & 0,33 & 0,49 & & 0,15 \\
\hline Cryptochironomus sp. & & & & 0,10 & & & & 0,03 \\
\hline Dicrotendipes sp. & & & & & 1,65 & & 1,50 & 0,55 \\
\hline Goeldichironomus neop. & 73,27 & 86,46 & 89,25 & 94,31 & 89,78 & 92,12 & 92,86 & 90,94 \\
\hline Parachironomus sp. & 16,83 & 7,29 & 4,45 & 2,69 & 6,26 & 4,68 & 2,63 & 4,75 \\
\hline Phaenopsectra sp. & & & & & 0,11 & 0,25 & 0,38 & 0,09 \\
\hline Polypedilum (Poly.) sp. & & & 0,15 & 0,10 & & & & 0,06 \\
\hline Endotribelos sp. & & & & & 0,22 & & & 0,06 \\
\hline Tanytarsini & 3,96 & & 1,23 & 0,50 & 0,22 & 0,99 & 1,13 & 0,76 \\
\hline Caladomyia ortoni & & & & 0,20 & & & & 0,06 \\
\hline Tanytarsus sp. & 3,96 & & 1,23 & 0,30 & 0,22 & 0,99 & 1,13 & 0,70 \\
\hline Tanypodinae & 4,95 & 4,17 & 4,15 & 1,30 & 1,10 & 1,23 & 0,75 & 1,92 \\
\hline Macropelopiini & & & & 0,10 & & & & 0,03 \\
\hline Fittkaumyia sp. & & & & 0,10 & & & & 0,03 \\
\hline Pentaneurini & 4,95 & 4,17 & 4,15 & 1,20 & 1,10 & 1,23 & 0,75 & 1,89 \\
\hline Ablabesmyia (Karelia) sp. & & 3,13 & 2,76 & 0,60 & 0,88 & 0,74 & 0,38 & 1,14 \\
\hline Labrundinia sp. & 2,97 & & 0,77 & 0,10 & 0,11 & & & 0,29 \\
\hline Larsia sp. & 1,98 & 1,04 & 0,61 & 0,50 & 0,11 & 0,49 & 0,38 & 0,47 \\
\hline Orthocladiinae & 0,99 & 1,04 & & 0,10 & 0,11 & & 0,38 & 0,15 \\
\hline Cricotopus sp. & & 1,04 & & & & & & 0,03 \\
\hline Nanocladius sp. & 0,99 & & & 0,10 & 0,11 & & 0,38 & 0,12 \\
\hline Total de indivíduos & 101 & 96 & 651 & 1002 & 910 & 406 & 266 & 3432 \\
\hline Total de táxons & 6 & 6 & 8 & 12 & 12 & 8 & 9 & 17 \\
\hline
\end{tabular}

TN: tela de náilon; I: isopor; P: pedra de argila expandida; A: areia grossa; M: madeira; S.c.: Scirpus cubensis; C.p.: Cabomba piauhyensis 

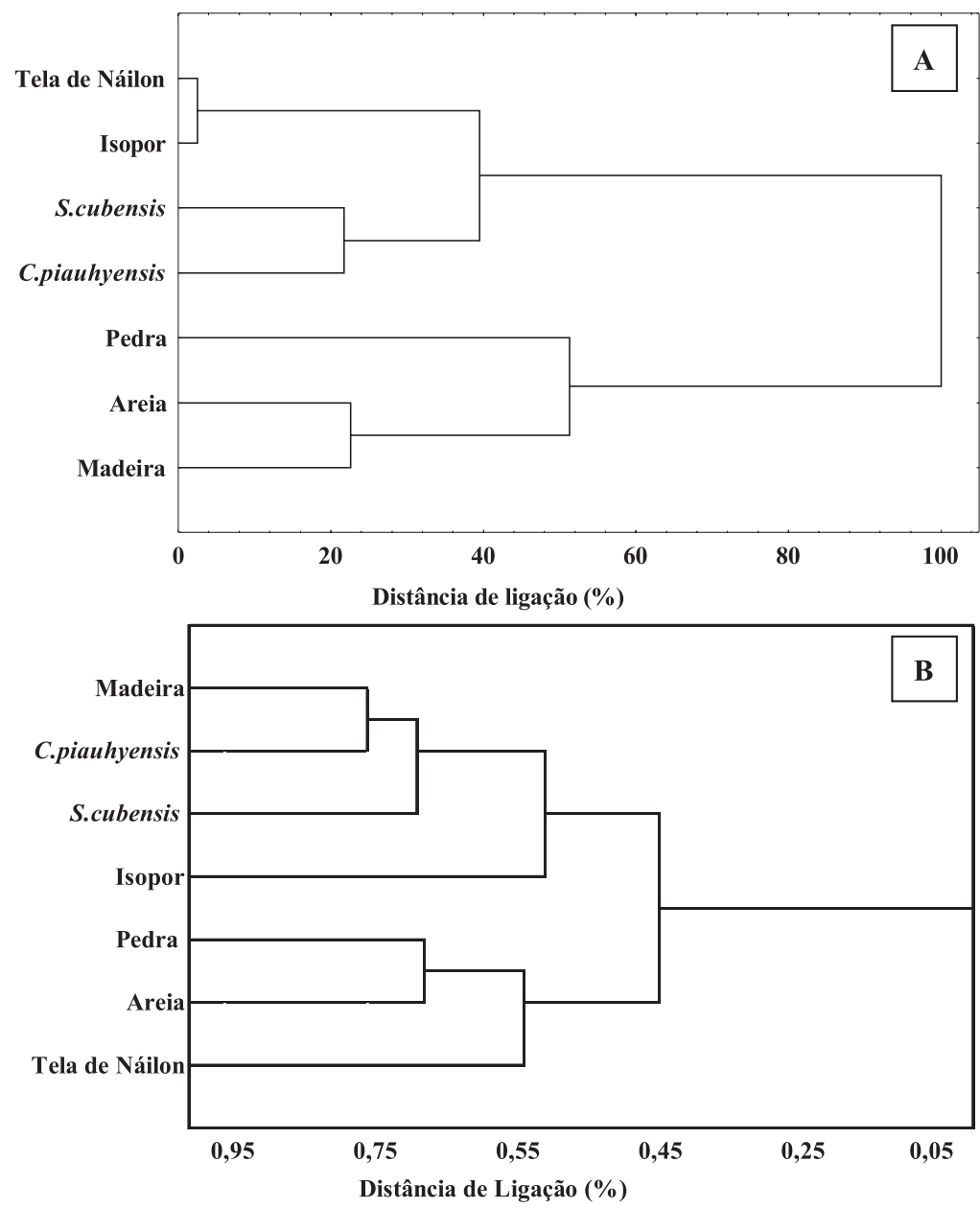

Figura 1: A: Dendograma de agrupamento das assembléias de Chironomidae nos substratos artificiais segundo a análise quantitativa (Distância Euclidiana); B: Dendograma de agrupamento das assembléias de Chironomidae nos substratos artificiais segundo a análise qualitativa (Coeficiente de Jaccard).

Devido à elevada participação de organismos coletores em todos os substratos (Fig. 2), as assembléias apresentaram-se funcionalmente similares. Digna de nota é a presença, embora reduzida, de quironomídeos raspadores nos substratos orgânicos vegetais. 


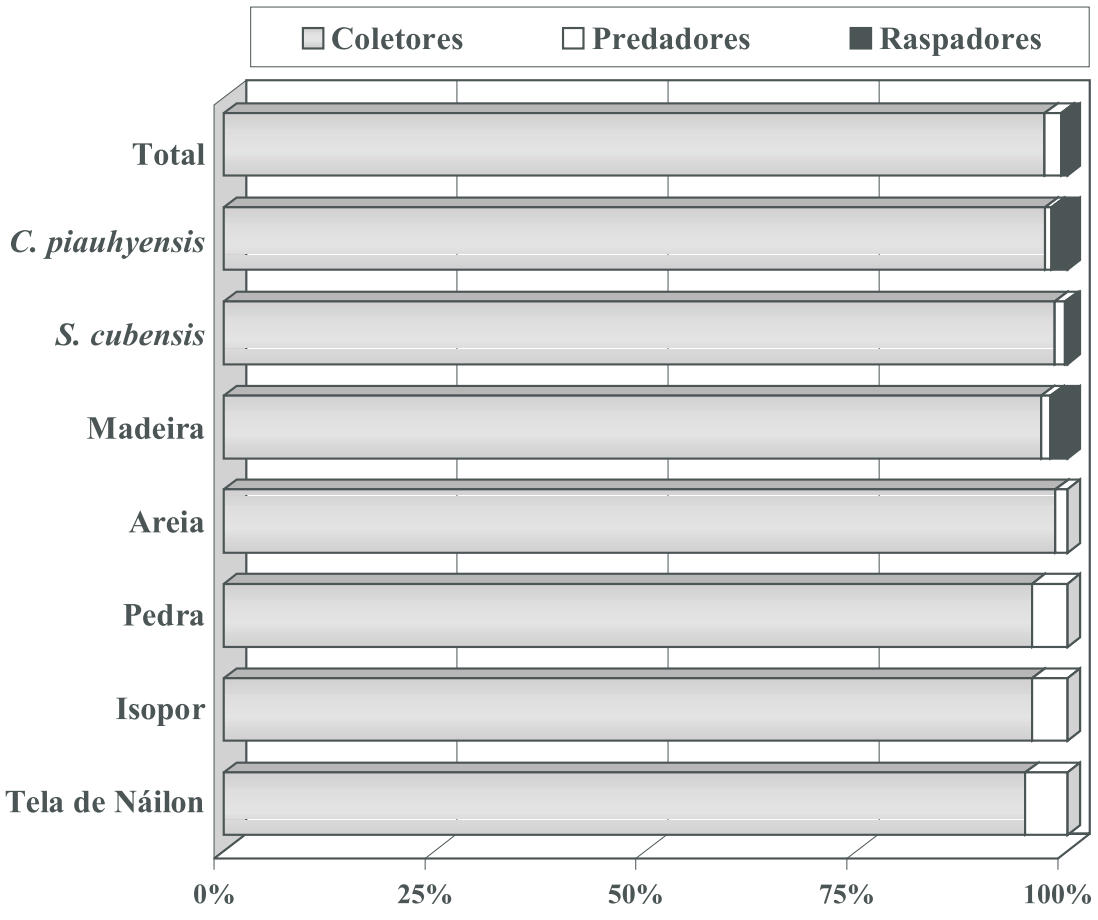

Figura 2: Participação relativa (\%) das guildas de alimentação das assembléias de Chironomidae presentes nos diferentes substratos artificiais.

\section{Discussão}

Considerando o predomínio de quironomídeos coletores em todo o experimento, pode-se supor que todos os substratos favoreceram a colonização por indivíduos desta guilda alimentar. Segundo Stockley et al. (1998), os sacos de colonização funcionariam como mini-barreiras capazes de reter detritos, quando presentes em grandes quantidades no sistema aquático. No presente estudo a prevalência de coletores pode, portanto, estar indicando que os sacos de colonização propiciaram o acúmulo de detritos em quantidade suficientemente atrativa à fauna. 
A possível preferência dos quironomídeos raspadores pelos materiais orgânicos vegetais (detritos de madeira e de macrófitas) é explicada considerando-se que o perifíton, principal alimento desses organismos, cresce com maior intensidade em substratos orgânicos em processo de decomposição (Oertli \& Lackavanne, 1995).

O processo de decomposição também parece ter influenciado a colonização dos diferentes substratos orgânicos. Ao contrário das observações feitas por diferentes autores (Imbert \& Pozo, 1989; Neiff \& Poi De Neiff, 1990; Bruquetas De Zozaya \& Neiff, 1991), foi notificado, no presente estudo, que as densidades numéricas dos Chironomidae nos substratos orgânicos (Madeira $>S$. cubensis $>C$. piauhyensis) foram inversamente proporcionais às suas velocidades de decomposição (Madeira < S. cubensis < C. piauhyensis). Estes resultados, no entanto, concordam com Oertli (1995) que relaciona as densidades de organismos com a superfície disponível do substrato.

Os substratos inorgânicos, apesar da similaridade demonstrada pela análise de agrupamento qualitativa, apresentaram diferenças que se assemelham aos resultados obtidos por Parker (1989). O substrato areia foi colonizado por uma maior densidade numérica de Chironomidae e maiores números de táxons e de indivíduos coletores, indicando que este substrato mineral de partículas menores acumularam maior quantidade de detritos pequenos (FPOM).

A despeito da expressiva participação de G. neopictus em todos os substratos, as análises de agrupamentos apontaram para maiores similaridades taxonômicas entre determinadas assembléias, embora a prevalência de quironomídeos coletores tenham tornado todas assembléias funcionalmente similares.

\section{Agradecimentos}

Somos gratos aos técnicos do Departamento de Hidrobiologia da UFSCAR, companheiros de laboratório e amigos, que auxiliaram em coletas e/ou triagem. 


\section{Referências Bibliográficas}

Bruquetas De Zozaya, I.Y. \& Neiff, J.J., 1991. Decomposition and colonization by invertebrates of Typha latifolia L. litter in Chaco cattail swamp (Argentina). Aquat. Bot. 40:185-193.

Cairns, J., 1982. Artificial substrates. Ann. Arbor Science Publishers Inc., Ann Arbor, Michigan. 279 p.

Cover, E.C. \& Harrel, R.C., 1978. Sequences of colonization, diversity, biomass, and productivity of macroinvertebrates on artificial substrates in a freshwater canal. Hydrobiologia 59:81-95.

Imbert, J.B. \& Pozzo, J., 1989. Breakdown of four leaf litter species and associated fauna in a Basque Country forested stream. Hydrobiologia 182:1-14.

Merritt, R.W. \& Cummins, K.W., 1984. An introduction to the aquatic insects of North America. 2nd ed. Kendall Hunt Publishing Co., Dubuque. 722 p.

Minshall, G.W., 1984. Aquatic insect-substratum relationships, pp. 358-400. In V. H. Resh \& D. M. Rosenberg (eds), The ecology of aquatic insects. Praeger, New York.

Neiff, J.J. \& Poi De Neiff, A., 1990. Litterfall, leaf decomposition and litter colonization of Tessaria integrifolia in the Paraná River floodplain. Hydrobiologia 203:45-52.

Oertli, B., 1995. Spatial and temporal distribution of the zoobenthos community in a woodland pond (Switzerland). Hidrobiologia 300/ 301:195-204.

Oertli, B. \& Lachavanne, J.B., 1995. The effects of shoot age on colonization of an emergent macrophyte (Typha latifolia) by macroinvertebrates. Freshwater Biol. 34: 421-431. 
Parker, M.S., 1989. Effect of substrate composition detritus accumulation and macroinvertebrate distribution in Southern Nevada Desert Stream. Southwest. Nat. 34:181-187.

Peso, J.G., 1995. Zoobentos del Arroyo Uruguai. Estudio preliminar (Missiones, Argentina). Rev. Asoc. Cienc. Nat. Litoral 26:21-37.

Stockley, R.A.; Oxford, G.S. \& Ormond, R.F.G., 1998. Do invertebrates matter? Detrital processing in the River Swale-Ouse. Sci. Total Environ. 210/211:427-435.

Street, M. \& Titmus, G., 1982. A field experiment on the value of allochthonous straw as food and substratum for lake macro-invertebrates. Freshwater Biol. 12:403-410.

Ward, J.V., 1992. Aquatic insect ecology I. Biology and Habitat- John Wiley and Sons. $450 \mathrm{p}$. 